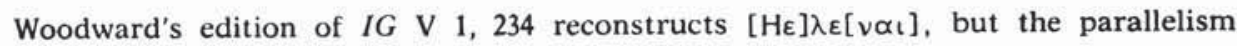
with other inscriptions of Spartan Meneleon suggests to reconstruct $\left[F_{\varepsilon}\right] \lambda \varepsilon[v \propto 1]$.

Cuatro inscripciones laconias, todas ellas antiguas y escritas en alfabeto epicórico, procedentes del Meneleon en Esparta, presentan muy probablemente una serie de dedicatorias a Helena ${ }^{1}$. De éstas solamente una ofrece una lectura clara de este nombre, las otras han sido reconstruidas.

1. SEG XXVI 458. Siglo vI:

$$
\tau \propto \iota \mathrm{F} \varepsilon \lambda \varepsilon \nu \propto \iota
$$

Escrita de derecha a izquierda.

2. SEG XXVI 457. Siglo VII-VI ${ }^{2}$ :

$$
\Delta \varepsilon เ v i[\varsigma]) \tau \propto \delta \propto v \varepsilon \theta \varepsilon \kappa \varepsilon X \alpha \rho ![.]) ~ F \varepsilon \lambda \varepsilon v a \underline{!} ! M \varepsilon v \varepsilon \lambda \propto F o)
$$

Escrita en bustrofedón.

3. SEG XXVII 407. Siglo vI:

$$
F \varepsilon[---]
$$

4. IG V 1, 234. Siglo VI:

$$
[H \in \varepsilon] \lambda \varepsilon[v \propto \mathrm{l}]
$$

1 No hemos incluido la inscripción $I G \mathrm{~V} 1,235$, donde también podría leerse una dedicatoria a Helena, ya que esta inscripción es más reciente (año 400 según A. M. Woodward, An. Br. Sc., 1909, p. 87).

2 Editadas ésta y la anterior por H. W. Catling y H. Cavanagh, Kadmos 15, 1976, pp. 145-152.

LIV, $2.0-8$ 
C. de Simone, en el artículo que dedica al estudio del nombre de Helena ${ }^{3}$, se basa precisamente en las dos inscripciones que hemos numerado como 1 y 2 para afirmar que el nombre ' $E \lambda \varepsilon \dot{v} \nu$ presentaba en laconio una $F$ inicial frente a la forma corintia $H \varepsilon \lambda \varepsilon \dot{\varepsilon} \alpha$ y a la calcidia $\mathrm{H} \varepsilon \lambda \varepsilon \dot{\varepsilon} v \eta$, atestiguadas también hacia el siglo vI.

La inscripción número 3 (siempre según nuestra numeración), descubierta aún más recientemente que las dos anteriores, presenta un inicial $F \varepsilon\left[\right.$, por lo que sus editores ${ }^{4}$ han supuesto una forma $F \varepsilon[\lambda \varepsilon v \alpha$.

La última, perteneciente a los descubrimientos hechos en el Meneleon en 1909, fue reconstruida por su editor, A. M. Woodward ${ }^{5}$, como $[H \varepsilon] \lambda_{\varepsilon}\left[v \alpha_{L}\right]$. Es evidente que, cuando esta reconstrucción fue hecha, se desconocía la existencia de las tres inscripciones ya citadas y que, por tanto, fue basada en las formas que este nombre tenía en otros lugares de Grecia o en las fuentes literarias. Creemos que hoy, gracias a la comparación con los testimonios de la misma época aproximadamente donde el nombre de Helena presenta con bastante seguridad una forma $F \varepsilon \lambda \varepsilon \dot{\varepsilon} \propto \alpha$, podemos afirmar que la reconstrucción más adecuada para la inscripción número 4 sería $[F \varepsilon] \lambda \varepsilon\left[v_{\alpha l}\right]$. Si en los siglos posteriores este nombre conservó esta forma con la digamma inicial, no podríamos decirlo con absoluta firmeza, aunque, dado que el dialecto laconio conservó siempre la digamma sobre todo en inicial, no vemos razón para que en este caso ésta fuera reemplazada por la aspiración.

\section{Mercedes Aguirre Castro}

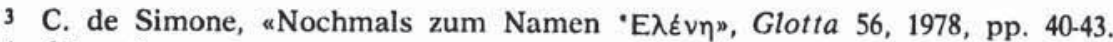

4 Cf. n. 2.

5 A. M. Woodward, An. Br. Sc., 1909, p. 86. 